\title{
Newsworthy neutrinos
}

\author{
The analysis of the KATRIN Collaboration's latest measurement campaign constrains the mass of the elusive \\ neutrino with unprecedented sensitivity.
}

A ccording to Frederick Reines and Clyde L. Cowan Jr, "each new discovery of natural science broadens our knowledge and deepens our understanding of the physical universe; but at times these advances raise new and even more fundamental questions than those which they answer. ${ }^{1}$ " Although referring to beta decay, they could have said this just as well about their discovery of the neutrino.

They gave the mass of the electron antineutrino to be less than " $1 / 500$ of the electron mass, if any" - with an emphasis on "if any". Around 40 years later, the observation of neutrino oscillations by the Super-Kamiokande ${ }^{2}$ and the Sudbury Neutrino Observatory ${ }^{3,4}$ collaborations gave a conclusive answer to this question: neutrinos oscillate, and therefore they have mass.

In addition to lower bounds from oscillations, the mass of neutrinos can be determined by different methods. Cosmological probes, such as the temperature anisotropy of the cosmic microwave background or galaxy clustering, constrain the sum of the three mass values. In addition, limits can be extracted from

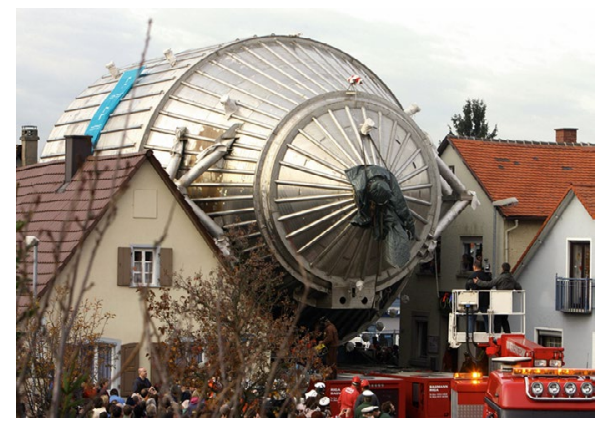

Credit: Michael Latz / DDP / AFP via Getty images

the half-life in searches for neutrino-less double-beta decay, and one can also gain information about the neutrino mass from beta decay kinematics because the endpoint of the decay spectrum is sensitive to the presence of the neutrino.

In this issue of Nature Physics, the KATRIN Collaboration reports ${ }^{5}$ a direct measurement of the effective electron antineutrino mass from the beta decay of tritium, constraining it to below $0.9 \mathrm{eV} c^{-2}$ at
90\% confidence level. Combined with their previous results ${ }^{6}$, the upper limit is further improved to $0.8 \mathrm{eV} \mathrm{c}^{-2}$. In the accompanying News \& Views, Angelo Nucciotti describes how challenging neutrino mass measurements are ${ }^{7}$.

Although the measurement by the KATRIN Collaboration advances our understanding of neutrinos, the words of Reines and Cowan still ring true: "the problem of detecting these cosmic end-products of all nuclear energy generation processes and the measurement of their characteristics presents a great challenge to the physics of to-day ${ }^{1}$."

Published online: 14 February 2022 https://doi.org/10.1038/s41567-022-01531-0

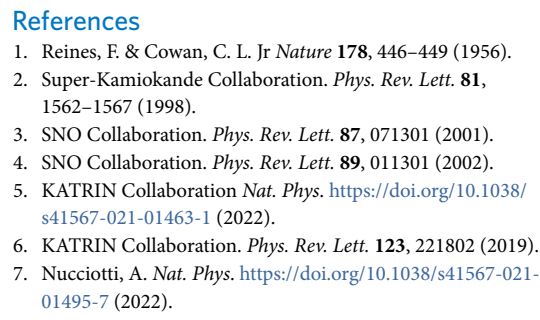

\section{Good to see you again}

As in-person conferences begin to make a return, we look forward to the opportunity to reconnect.

$\mathrm{T}$ wo years into a global pandemic, we have witnessed a breathtaking achievement by the world's scientific community. They have understood how COVID-19 is transmitted, developed a range of highly effective vaccines, and created efficient therapies for fighting the disease. This success means that some features of 'normal life' are starting to resume, and in-person conferences are one of those.

For the last two years, scientific collaboration has been forced online. There are certainly upsides to virtual meetings: the reduced carbon footprint and ease of access being two obvious ones. But even the best virtual space still lacks the subtle joy of meeting old friends and making new acquaintances in person, and it is only natural that many scientists are looking forward to travelling again.
Both the SPIE and APS have re-introduced a large in-person component to their flagship events Photonics West and the March and April meetings, respectively. Attendees are required to be fully vaccinated and wear a mask at all times so the risk of transmission is reduced as much as is practical.

Like many researchers, our editorial team views conferences as an indispensable way of connecting with the community, keeping up with what excites our readers, receiving feedback, and igniting ideas for projects and commissioning. We have sorely missed the serendipitous conversations over a coffee break or a meal, and the more candid opinions that these informal interactions can encourage. We are therefore very much looking forward to reconnecting, and this will start at the March Meeting in a few weeks.

As we have noted previously (Nat. Phys. 17, 287; 2021), online and hybrid conferences are here to stay and we should continue improving the experience that they provide. One particular challenge seems to be managing the combination of in-person and remote attendance, so that those not at the venue can participate fully. Now is the moment to test new ideas, find out what works, and move on from what doesn't. By doing so, we can retain the best of both modes of meeting, and build a more accessible and productive future for scientific collaboration.

Published online: 14 February 2022 https://doi.org/10.1038/s41567-022-01530-1 